HEAD TRAUMA AND COMA

HEAD INJURY IN INFANTS AND CHILDREN

Of 738 children with head injuries (0-16 yrs) admitted to the Children's Memorial Hospital, Chicago, IL, during a 5-year period from 1981-85, 318 (43\%) were less than 3 years of age and almost half of the younger group were under 1 year. A fall was the most common mechanism of injury in children under 3 years, followed by a motor vehicle accident. Post-traumatic seizures developed more commonly in children under 2 yrs (16\%) than in older children (98, entire group). The most reliable indicators of poor outcome were absent or impaired oculovestibular reflex and bilateral fixed dilated pupils. Intracranial pressure greater than 40 torr with coma scores of 3,4 or 5 spelled fatality. The mortality of the entire group with severe head injury was 22\%. (Hahn YS et al. Head injuries in children under 36 months of age. Demography and outcome. Child's Nerv Syst Feb $1988 ; \underline{4}: 34-40$ ).

COMMENT: A modified Glasgow Coma Scale -- Children's Coma Scale -- was developed as an objective neurological assessment and prognostic indicator for the children under 3 yrs of age in this study. Points for eye-opening responses (4-1) were the same but those for best motor response and best verbal response were different for infants. Smiling, orientation to sound or verbal stimulus, or following objects were given a subscore of "5" (oriented); consolable crying but inappropriate interaction "4"; inconsistent consolable crying and moaning " 3 ; inconsolable crying and irritable, restless interaction " 2 "; no response " 1 ". One third of the younger group showed a sequence of labile symptoms following the initial loss of consciousness, becoming agitated and irritable, followed by vomiting, sleep, and waking and playful within $24 \mathrm{hrs}$ in the majority. When children were comatose longer than $6 \mathrm{hrs}, 75 \%(12 / 16)$ had a poor outcome with a $50 \%(8 / 16)$ mortality.

\title{
CONSCIOUS LEVEL ASSESSMENT IN INFANTS
}

Neurosurgeons at the Adelaide Children's Hospital, King William Street, North Adelaide, SA 5006, Australia have used a paediatric version of the Glasgow Coma Scale since 1977 for assessing conscious level in infants and young children. For the best verbal response, during the first 6 months the normally conscious infant is expected to cry or grunt spontaneously or when disturbed and the expected normal score is 2 . Between 6 and 12 months, the normal infant babbles and begins to vocalize and scores 3. After 12 months, words are expected with a score of 4 . Orientation by 5 years of age gives a score of 5 . The normal aggregate scores at different ages are as follows: birth-6 mth: 9; 6-12 mth: 11; 12-24 mth: 12; 2-5 yr: 13; over 5 yr:14. A disadvantage of the scale is the reduced sensitivity, especially in the neonate. (Reilly PL et al. Assessing the conscious level in infants and young children: a paediatric version of the Glasgow Coma Scale. Child's Nerv Syst 1988; 4 :30-33).

COMMENT: In an editorial comment, Dr AJ Raimondi corroborates the need for children's and infant's coma scales as substitutes for the well known Glasgow scale suitable mainly for adults. He favors an 
1l-point scale for infants, which incorporates ocular responses (ranging from normal pursuit to fixed pupils), verbal responses (ranging from crying to apnea), and motor responses (from flexion/ extension to flaccidity). A uniformly tested and accepted coma scale for infants and children would be advantageous. Coma scores as low as 3-4 carry the same prediction of poor outcome at any age; coma scores of 5-8 may carry a less serious significance in young children than in adults.

\section{ALPHA COMA IN THE EEG}

Recorded EEG rhythms within the alpha-frequency band, paradoxically resembling waking patterns but in apparently comatose patients, a pattern termed alpha coma, have been reviewed over a $10 \mathrm{yr}$ period in the Division of Electroencephalography, University of Washington School of Medicine, Seattle, WA. Of 50 patients with records showing the alpha-pattern coma, 49 were admitted with cardio-pulmonary arrest and one had developed alpha coma with hyperglycemic, hyperosmolar coma. In addition to the alpha activity, arrhythmic delta waves were present diffusely in $25 \%$ and theta waves in 23\%. The outcomes of patients with or without alpha coma after cardiac arrest did not differ significantly, the majority not regaining consciousness and dying in hospital. The single patient with hyperglycemic coma regained consciousness and was discharged but did not fully recover cognitive function. A review of the literature did not preclude neurological recovery following alpha coma. (Austin EJ et al. Etiology and prognosis of alpha coma. Neurology May 1988; 38: 773-777).

COMMENT: Alpha coma usually follows cardiac arrest and can be identified in $25 \%$ of patients. It also follows brainstem lesions, sedative drug overdose, respiratory arrest, and severe disturbance of glucose metabolism. Where alpha coma follows a condition other than cardiac arrest or a brain stem lesion the outcome is usually good. The prognosis is especially favorable after drug overdose.

\section{MOVEMENT DISORDERS}

\section{DYSTONIA AND BIOPTERIN DEFICIENCY}

Five patients with childhood dystonia associated with reduced CSF biopterin, responsive to levodopa, and characterized by diurnal and exertional variation are reported from the Developmental and Metabolic Neurology Branch, National Institute of Neurological and Communicative Disorders and Stroke, Bethesda, MD. Of 4 familial cases of dystonia with biopterin deficiency limited to the CNS and of unknown etiology, 2 brothers were more severely affected than 2 sisters, and the dystonia was complicated by hyperreflexia and extensor plantar responses indicative of associated pyramidal tract involvement. The fifth patient had a systemic deficiency of biopterin with hyperphenylalanemia and atypical phenylketonuria, and his dystonic rigidity was purely extrapyramidal and without spasticity. Patients were least symptomatic in the early morning or after a nap and became progressively weak, rigid and dystonic during the day with complete immobility in the afternoon or evening, and often unable to speak or to swallow. Improvement following Sinemet 10/100 began within 36 hours and benefit has been sustained during treatment for 18 